\title{
Effect of Different Parameters on Segmentation of Binary Images using Genetic Algorithms
}

\author{
B.D. Phulpagar, PhD \\ Computer Engineering Department \\ P. E. S. Modern College of Engineering, \\ Pune, India.
}

\author{
R.S. Bichkar, $\mathrm{PhD}$ \\ Electronics and Telecommunication Department \\ G. H. Raisoni College of Engineering and \\ Management, Pune, India.
}

\begin{abstract}
Segmentation is a process of separating the objects from background. Several GA-based approaches have been used to improve the quality of image segmentation in the past based on thresholds, edge detection, region and cluster detection and morphology. The proposed GA-based approach gives us good results for the segmentation of binary images using different image parameters namely the image block size $4 \times 4,8 \times 8$, and $16 \times 16$ pixels, the difference between the object and background intensities. Also the proposed approaches gives us best segmentation results for various GA parameters is considered that include population size (minimum 40), number of GA generations i.e. 80 and crossover rate more than 0.50 and less than or equal 0.70 and mutation rate between 0.01 and 0.08 . The results obtained give $100 \%$ pixel classification accuracy for Speckle noise with noise levels more than $2.70 \mathrm{~dB}$ SNR and $93 \%$ to $96 \%$ for salt and pepper noise levels (SNR ranging between $7.69 \mathrm{~dB}$ to $11.92 \mathrm{~dB}$ ). The segmentation results obtained by using proposed GA-based method are good with the increasing noise density of Speckle as compared to salt and pepper noises.
\end{abstract}

\section{General Terms}

Image Processing, Pattern Recognition.

\section{Keywords}

Genetic Algorithm, GA Parameters, Image Parameters and Image Segmentation.

\section{INTRODUCTION}

The traditional techniques of optimization generate a deterministic sequence of trial solutions. They are based on the gradient or higher order statistics of the cost function. In some cases they converge extremely fast. Some of the variations in the existing methods are applied to estimation of parameters in pattern recognition, image processing, segmentation of images, image enhancement, machine learning, expert system, adaptive image processing and adaptive control applications. But these methods often fail to perform effectively when applied to difficult problems. Genetic algorithm has emerged from a study of the mechanics of evolution. It is a stochastic optimization method that gives better results as compared to traditional optimization techniques when applied to difficult real world problems. The important characteristic of GA is that it is robust and can deal successfully with a wide range of problem areas.

Several researchers have used GA for image segmentation, which involves exploration of a huge search space. Bradhurst et al. [1] have presented a GA-based segmentation method. It uses an automatic thresholding algorithm to segment regions of high intensity variance form regions of low intensity variance. However, the presented algorithm does not segment an image properly if it has an insufficient background area.
Bosco [2] proposed a GA based method that considers the segmentation problem as a global optimization problem in which the fitness of GA individuals is calculated using the similarity between images. The quality of the segmented images is affected due to the natural changes in the image. Bhanu et al. [3] proposed a closed-loop image segmentation technique that incorporates a GA to adapt the segmentation process to changes in the image characteristics caused by variable environmental conditions. However, this method required appropriate image characteristics set to achieve adequate result. Bichkar and Ray [4] have proposed a method to detect the number of circular and elliptical objects and to estimate object parameters, namely the location, size and shape in given images. Haseyama et al. [5] have studies criterion like parameter of model, shape and location of the regions. Cornely et al. [6] have proposed GA-based medical image segmentation method. In this approach, have studies different parameters for image segmentation. Horita et al. [7] have proposed method to obtain region segmentation using kmean clustering and GAs. It show the region segmentation have been achieved by using parameters of GAs. Schroeter et al. [8] and Cinque et al. [9] have studies different parameters for GA-based segmentation techniques.

The existing methods motivated us to explore the effect of different parameters on segmentation of binary images using GAs and also in presence of noise.

In this paper, the GA-based method is implemented for evaluate the performance of GA for the segmentation of binary images using different GA parameters and also the effect of various image parameters. The results obtained show an improvement in segmentation of images using GA-based method with different parameters and appropriate value.

\section{IMAGE SEGMENTATION USING GA}

The goal of the proposed method for image segmentation is to divide an image into two classes the object and the background using different GA and image parameters. These are the regions that are homogeneous according to certain characteristics. The greay level of the pixels in the image is the characteristic considered for segmentation in the proposed method. The images considered in this method are corrupted by adding salt and pepper noise and Speckle noise. The SNR of images of size $M \times N$ is defined [10] as the ratio of average signal power to average noise power. SNR is mean-squared error measure and its unit is decibel $(\mathrm{dB})$.

$$
\operatorname{SNR}(d B)=10 \log _{10} \frac{\sum_{\mathrm{i}=1}^{\mathrm{M}} \sum_{j=1}^{\mathrm{N}} \mathrm{a}_{\mathrm{ij}}{ }^{2}}{\sum_{\mathrm{i}=1}^{\mathrm{M}} \sum_{\mathrm{j}=1}^{\mathrm{N}}\left(\mathrm{a}_{\mathrm{ij}}-\mathrm{b}_{\mathrm{ij}}\right)^{2}}
$$

Where $a_{i j}$ denotes $i j^{\text {th }}$ pixel of the original image and $b_{i j}$ denotes $i j^{t h}$ pixel of the noisy image $B$.

In this technique, a 2-D image block of size $m \times n$ is 
represented using binary vector of size $m \times n$ as, $\bar{P}=$ $\left[p_{1}, p_{2}, \ldots, p_{k}, \ldots, p_{m n}\right]$ where the position of a pixel in $i^{\text {th }}$ row and $j^{\text {th }}$ column is given as $k=(i-1) * n+j$, thus the GA chromosome is a $\mathrm{m} \times n$ sized bit string, with each $p_{k}$ representing a gene of any one of two constant greay classes. Here, the fitness function is a measure of similarity between a GA individual and the original noisy image. The fitness of each chromosome in the population for an image block of size $m \times n$ is defined by following equation

$$
\text { fitness }(\bar{P})=\frac{1}{1+\frac{1}{m * n} \sum_{i=1}^{m} \sum_{j=1}^{n}\left|b_{i j}-p_{k}^{\prime}\right|}
$$

Where $b_{i j}$ is an $\mathrm{ij}^{\text {th }}$ pixel in a block of input image, $p_{k}^{\prime}$ is the corresponding pixel value for gene $p_{k}$ in chromosome $\bar{P}$ and $k=(i-1) * n+j$. The initial population of GA is randomly generated and the fitness of each individual is calculated. Then successive generations improve it through repetitive applications of genetic operators namely, selection, crossover and mutation until the desired solution obtained. The population size is kept constant over the entire GA run.

The selection function is implemented by using the concept of biased roulette wheel [11], in which each individual is assigned a roulette slot in proportion to its fitness. Reproduction step consists of crossover and mutation operators [11]. The crossover operator uses one-point mechanisms. The mutation operator is implemented as a $3 \times 3$ median filter, where the central pixel in $n \times n$ window is replaced with the median of all the pixel values in the window. Finally the quality of image segmentation depends on different GA and image parameters.

\section{THE RESULTS AND DISCUSSIONS}

In this proposed work, an attempt has been made to segment the image into two distinct classes by genetic algorithm. The quality of segmented image is determined from the number of misclassified pixels between original image and segmented image where as the convergence speed of GA is determined from the fitness of the best GA individual of each GA generation. To understand the effect of various GA and image parameters on quality of image segmentation as well as on GA convergence, several experiments are performed and results are presented in this paper. The various parameters considered include GA parameters namely, number of GA generations, population size and crossover and mutation probabilities and image parameters namely, image block sizes, matching of object boundaries match with segmented block boundaries, type and density of noise and object and background intensities.

The results are presented using synthetic images Figure 1 (a) and (b) having two classes with pixel intensities 50 and 150 and Figure 1 (c) consists background and object with 0 and 80 pixel values respectively. The size of each image is $64 \times 64$ pixels. Each of them is called as "original image". The corrupted images are obtained by adding noise (salt and pepper or Speckle) with different densities to the original images. The image to be segmented is divided into nonoverlapping sub-images of size $4 \times 4$ pixels. Later segmented sub-images are combined to obtain the entire segmented image. Here, the chromosomes are 16-bit strings.

A simple GA was first used for the segmentation of noiseless images. An attempt is made to study the effect of variations in these parameters in order to identify suitable parameters for image segmentation application and achieve good results in minimum execution time. In each GA run, variation in one particular parameter is considered with other parameters being constant. We have first tested the effect of number of GA generations, population size, and crossover and mutation probabilities.

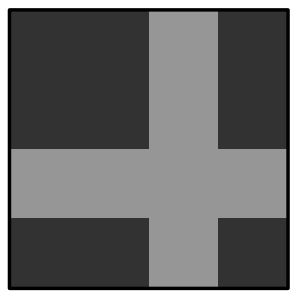

(a)

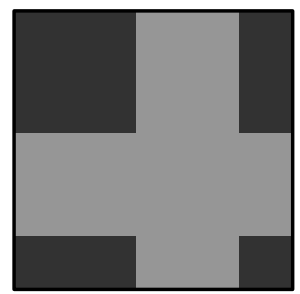

(b)

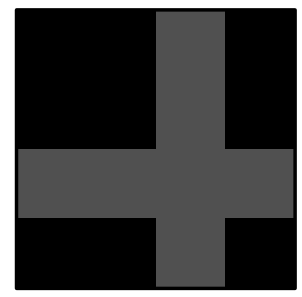

(c)
Figure 1: Set of synthetic images.

Effect of number of GA generations: GA parameter set for these experiment are as follows: Population size $=40$, Number of GA generations $=($ at 5, 20, 40,60, 80 and 100), Mutation probability $=0.01$, Crossover probability $=0.6$, Mutation operator: Bit-Flip and Crossover operator: onepoint.

Figure 2 (a) - (f) shows the results obtained using proposed GA at different number of generations (after 5, 20, 40, 60, 80 and 100 generations). It may be observed that the error in the segmentation output reduces as the GA run progresses and after 80 generations, the error in the segmentation is zero.

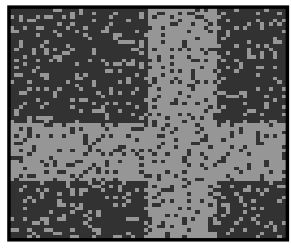

(a)

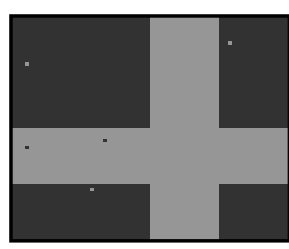

(d)

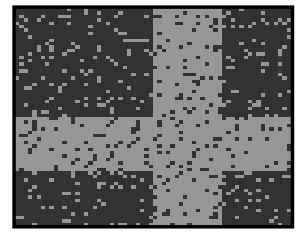

(b)

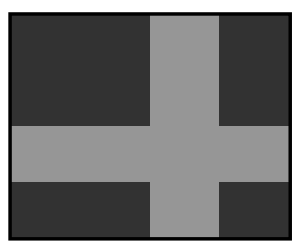

(e)

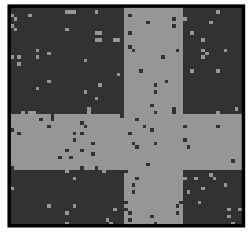

(c)

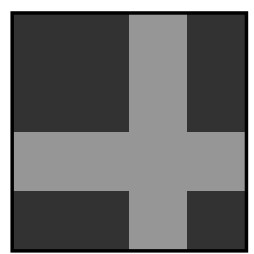

(f)
Figure 2: Segmentation results obtained by GA for Figure 1 (a) after 5, 20, 40, 60, 80 and 100 generations.

Table 1 shows the misclassified pixels and the segmentation accuracy as percentage of classified pixels for these images.

Table 1: Segmentation accuracy using GA for GA run depicted in Figure 2.

\begin{tabular}{|l|l|c|c|c|}
\hline $\begin{array}{l}\text { Sr. } \\
\text { No. }\end{array}$ & $\begin{array}{l}\text { Segmented } \\
\text { Image }\end{array}$ & $\begin{array}{l}\text { Number of } \\
\text { Generations }\end{array}$ & $\begin{array}{l}\text { Misclassified } \\
\text { Pixels }\end{array}$ & $\begin{array}{l}\text { Segmentation } \\
\text { Accuracy (\%) }\end{array}$ \\
\hline 1 & Figure 2 (a) & 05 & 712 & 82.8 \\
\hline 2 & Figure 2 (b) & 20 & 456 & 88.9 \\
\hline 3 & Figure 2 (c) & 40 & 139 & 96.6 \\
\hline 4 & Figure 2 (d) & 60 & 005 & 99.9 \\
\hline 5 & Figure 2 (e) & 80 & 000 & 100.0 \\
\hline 6 & Figure 2 (f) & 100 & 000 & 100.0 \\
\hline
\end{tabular}

Effect of population size: Population size is important 
parameters of GA. It decides the quality of output and run time of the algorithm. In order to ensure that GA converges to the global solution, the population size must be large. The parameter set for these GA runs is as follows: Population sizes $=10,20,30$ and 40, Number of generations $=40$, Mutation probability $=0.01$, Crossover probability $=0.6$, Mutation operator: Bit-Flip and Crossover operator: one-point. Figure 3 shows the results obtained of these GA runs. Table 2 shows the misclassified pixels and the segmentation accuracy for these GA runs. It is clear that larger GA population size gives better results.

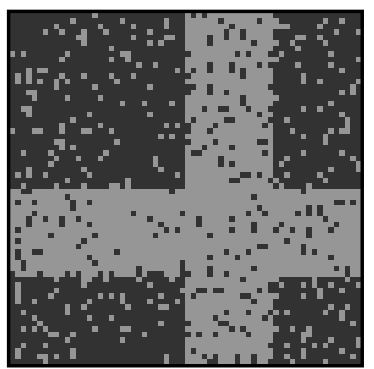

(a)

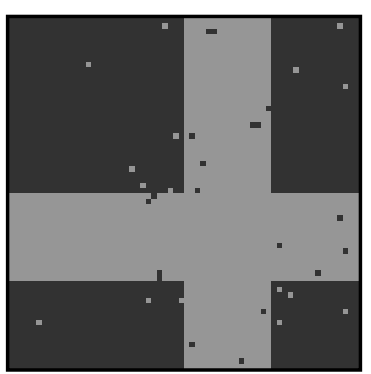

(c)

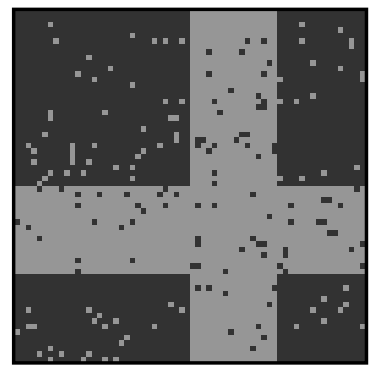

(b)

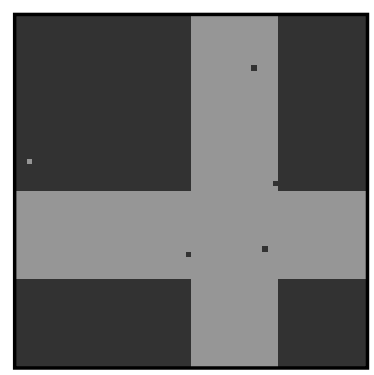

(d)
Figure 3: (a)-(d) Segmentation results obtained by GA for input image of size $64 \times 64$ shown in Figure 1 (a) for GA population sizes of 10, 20,30 and 40 respectively, after 40 generations.

Table 2: Effect of GA population size on the accuracy of segmentation of two-class image of size $64 \times 64$ using GA.

\begin{tabular}{|c|l|r|c|c|}
\hline $\begin{array}{c}\text { Sr. } \\
\text { No. }\end{array}$ & $\begin{array}{l}\text { Segmented } \\
\text { Image }\end{array}$ & $\begin{array}{c}\text { GA } \\
\text { Population } \\
\text { Size }\end{array}$ & $\begin{array}{c}\text { Misclassified } \\
\text { Pixels }\end{array}$ & $\begin{array}{c}\text { Segmentation } \\
\text { Accuracy (\%) }\end{array}$ \\
\hline 1 & Figure 3 (a) & 10 & 472 & 88.4 \\
\hline 2 & Figure 3 (b) & 20 & 178 & 95.6 \\
\hline 3 & Figure 3 (c) & 30 & 37 & 99.1 \\
\hline 4 & Figure 3 (d) & 40 & 5 & 99.8 \\
\hline
\end{tabular}

Effect of crossover and mutation operators: Other GA parameters are crossover and mutation probabilities. The GA parameters were set as follows: Population sizes $=40$, Number of generations $=50$, Mutation operator: Bit-Flip and Crossover operator: one-point.

Figure 4 (a) shows that the proposed GA with crossover rate between 0.50 to 0.70 gives the $100 \%$ pixel classification accuracy for Figure 1 (a). Another observation is that the error is high in segmented images for crossover rate below 0.50 and above 0.70 .

Figure 4 (b) shows that the GA runs with mutation rates between 0.01 and 0.08 gives $100 \%$ pixel classification accuracy for Figure 1 (a). However, for other mutation rates it gives poor segmentation results.

Effect of image block sizes: The GA was used to explore the effect of parameters of blocks used for image segmentation. The segmentation depends on two factors namely block size and whether the block boundaries coincide with the object boundaries in the image. Figure 1 (a) shows original image in which the image edges coincided with block boundaries. GA was applied for segmentation of this image. Three different block sizes were used namely $4 \times 4,8 \times 8$, and $16 \times 16$.

The GA parameters were set as follows: Population sizes $=$ 40 , Number of generations $=100$, Mutation probability $=$ 0.01 , Crossover probability $=0.6$, Mutation operator: Bit-Flip and Crossover operator: one-point.

The GA convergence for these three runs is shown in Figure 5 . It may be observed that block size $4 \times 4$ gives much better result compared to the $8 \times 8$ and $16 \times 16$ block sizes.

Figure 1 (b) shows an image in which the object edges do not coincide with the block boundaries for blocks of size $4 \times 4$, $8 \times 8$, and $16 \times 16$. The GA was applied for segmentation of this image using block sizes of $4 \times 4,8 \times 8$ and $16 \times 16$. Figure 6 shows the results obtained. The $4 \times 4$ block size gives better results in this case as well. Also note that these GA runs require more generations for convergence.

Effect of noise on segmentation of images: Noise present in the image affects the quality of the image segmentation. The test image consists of two classes, background and object with 0 and 80 pixel values respectively. Noisy test images are obtained by adding different types of noise namely Speckle and salt and pepper to original image shown in Figure 1 (c). In this section, we study how the quality of segmented image is affected due to noise and noise level.

First, the results are presented for the segmentation of images containing Speckle noise. Noisy images are obtained by adding Speckle noise with density $0.02,0.04$ and 0.08 . These images have SNR of $4.37 \mathrm{~dB}, 3.19 \mathrm{~dB}$ and $2.70 \mathrm{~dB}$ respectively and are shown in Figure 7 (a) - (c). The GA parameters are as follows: Population sizes $=40,50$ and 60 , Number of generations $=200$, Mutation probability $=0.01$, Crossover probability $=0.6$, Mutation operator: Bit-Flip and Crossover operator: one-point. Also, $4 \times 4$ image block size is used for segmentation.

The segmented images are shown in Figure 7 (d) - (f). Figure 8 show the convergence graph of the GA run with $4 \times 4$ block size for variations in Speckle noise with $2.70 \mathrm{~dB}, 3.19 \mathrm{~dB}$ and $4.37 \mathrm{~dB}$ SNR. It may be observed that in all cases, the initial convergence is very rapid and after about 50 generations, there is no much reduction in the output error. It may be noted that although the segmented images have no misclassified pixels, the error in the GA run is quite high (few thousands) due to presence of noise in the noisy image provided to GA for segmentation.

Table 3 presents the results (the number of misclassified pixels and segmentation accuracy) for these GA runs. It is observed that there are no misclassified pixels in the segmented images i.e. the segmentation accuracy of $100 \%$ is achieved.

Next, the GA is applied for segmentation of Salt and Pepper noisy images shown in Figure 9 (a) - (c) having SNR of 11.92 $\mathrm{dB}, 8.57 \mathrm{~dB}$ and $7.69 \mathrm{~dB}$ respectively. The GA parameters are number of generations is set to 140 , Population sizes $=50,60$ and 70 and the other parameters are same as well. Figure 9 (a) - (c) show noisy input images and corresponding segmented images are shown in Figure 9 (d) - (f) respectively. Figure 10 
show the convergence graph of the GA run with $4 \times 4$ block size for variations in Salt and Pepper noise with $7.69 \mathrm{~dB}, 8.57$ $\mathrm{dB}$ and $11.92 \mathrm{~dB}$ SNR. It may be observed that in all cases, the initial convergence is very rapid and after about 50 generations, there is no much reduction in the output error.
Table 4 presents the results for these GA runs. It may be observed that the segmented images contain misclassified pixels and have the classification accuracies ranging from $93 \%$ to $96 \%$.

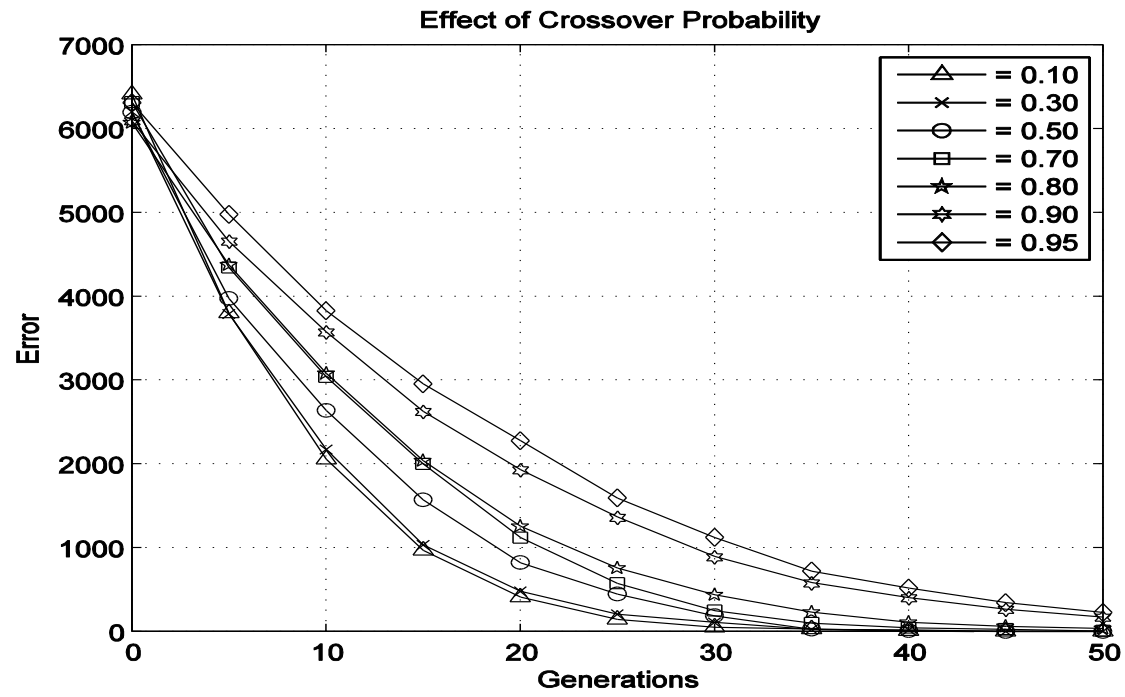

(a)

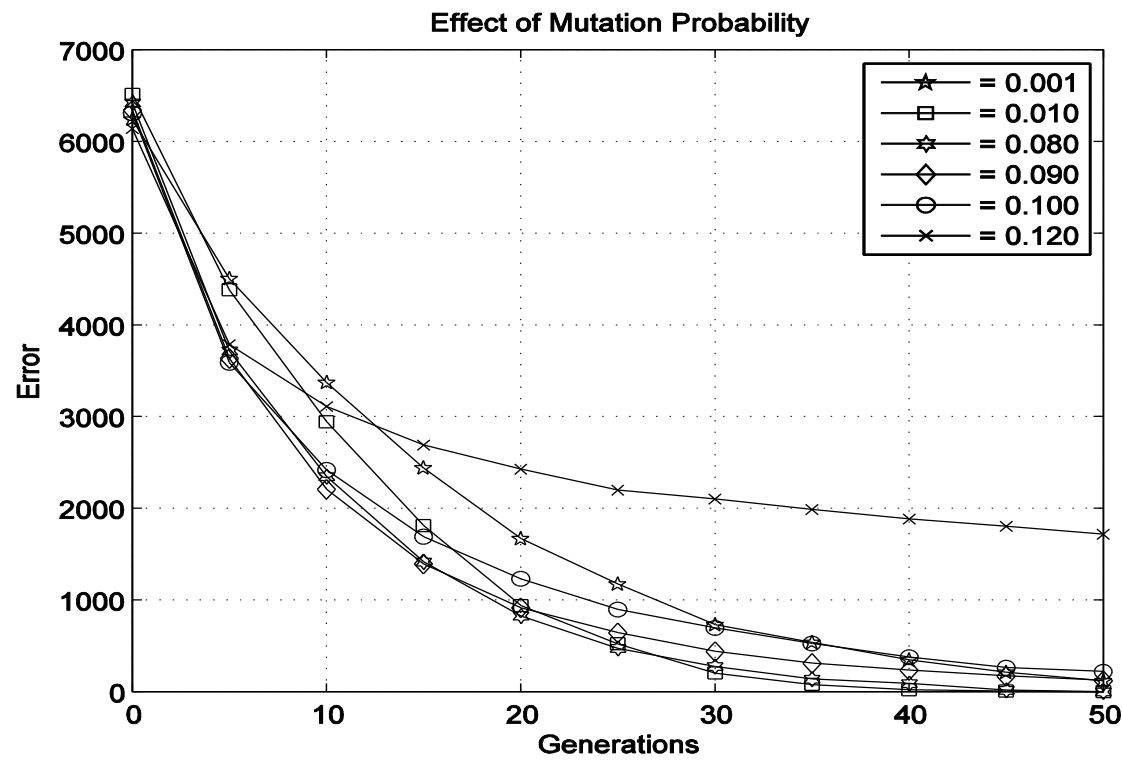

(b)

Figure 4: Convergence of GA runs at different crossover rates (a) and mutation rates (b) for image shown in Figure 1 (a). 


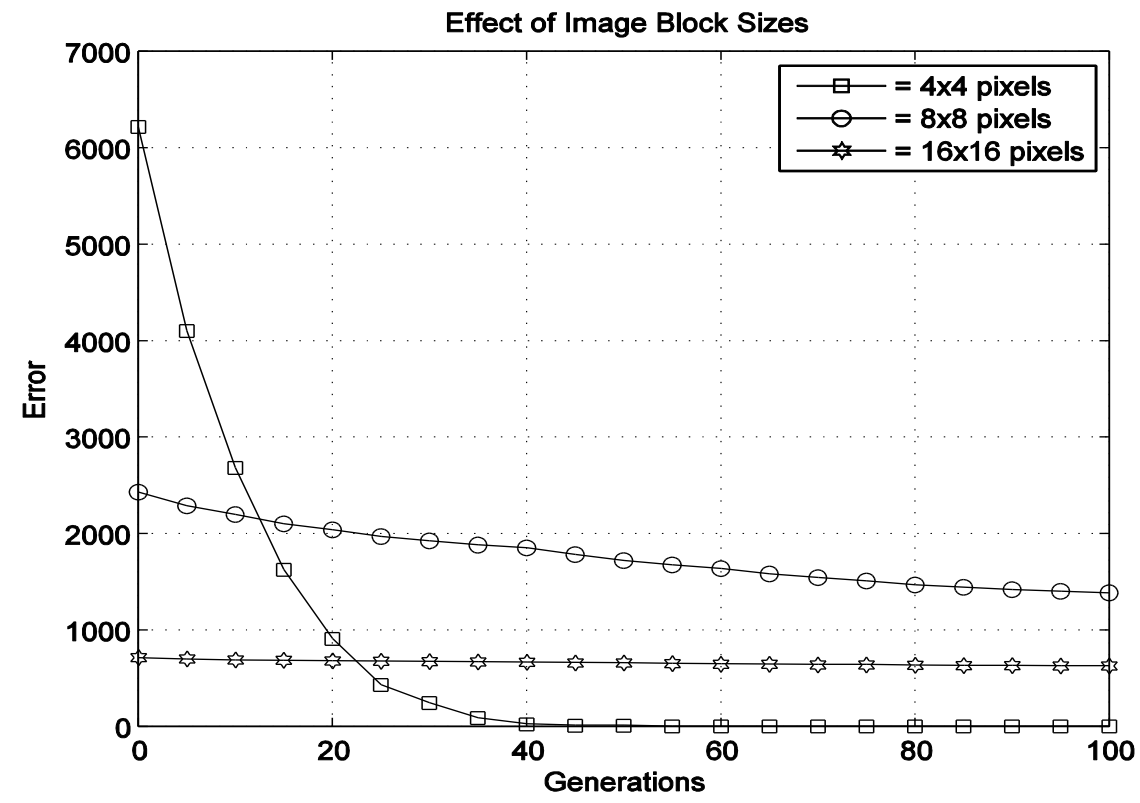

Figure 5: Convergence of GA runs using different block sizes for image shown in Figure 1 (a) in which block boundaries coincide the image edges.

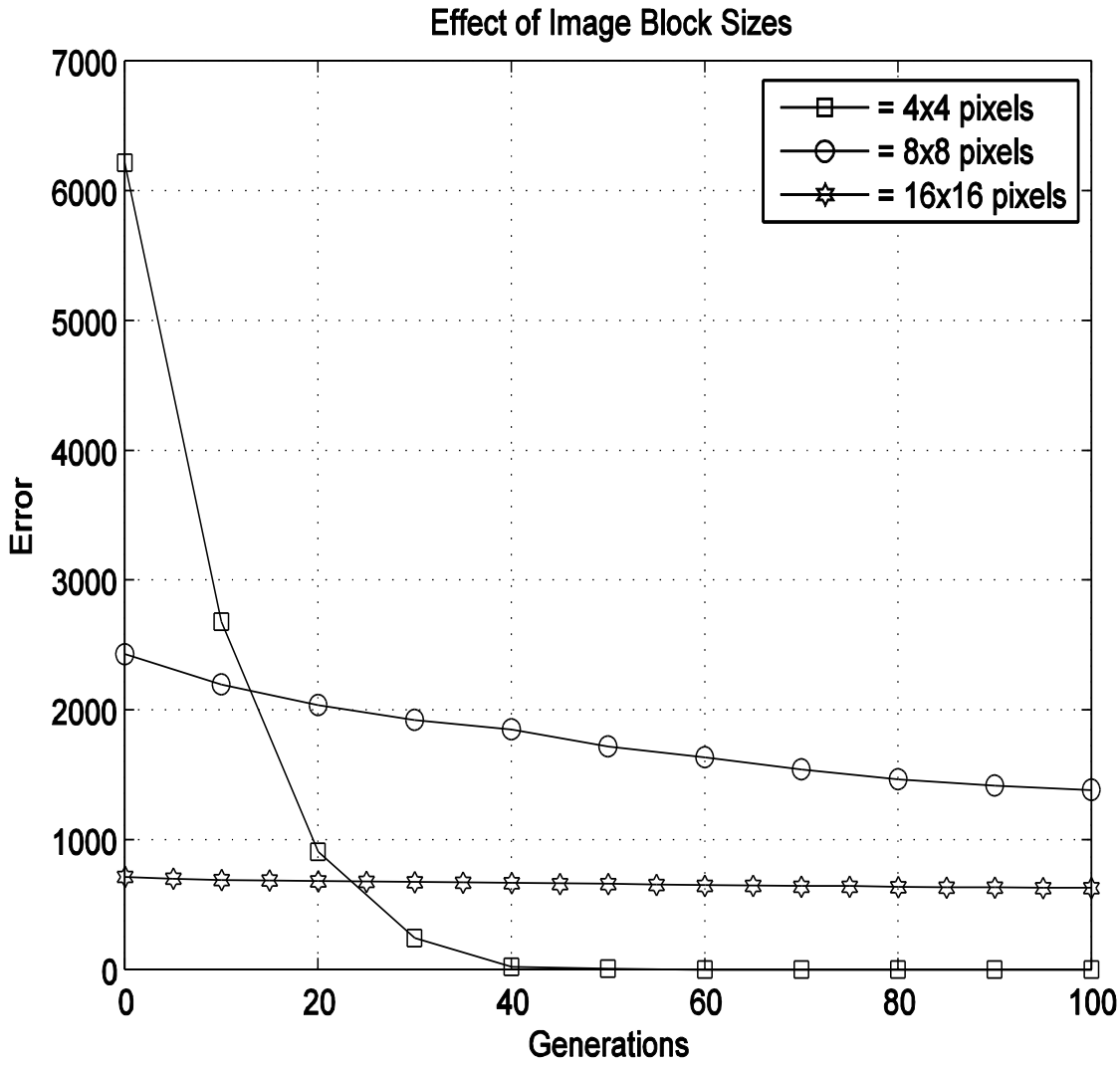

Figure 6: Results for segmentation of image in which image edges do not coincide the block boundaries Figure 1 (b) Original image and GA convergence for $4 \times 4,8 \times 8$ and $16 \times 16$ block sizes. 


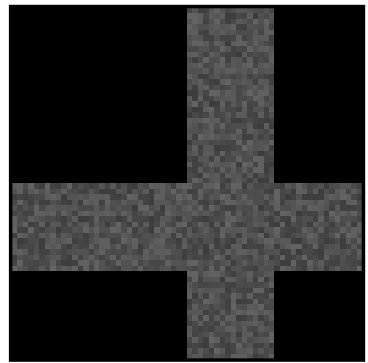

(a)

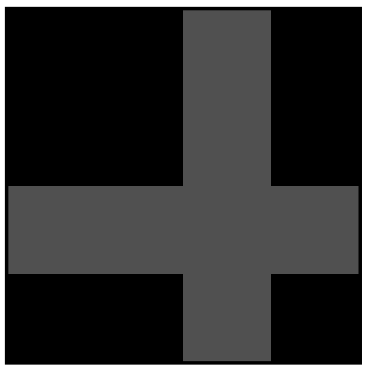

(d)

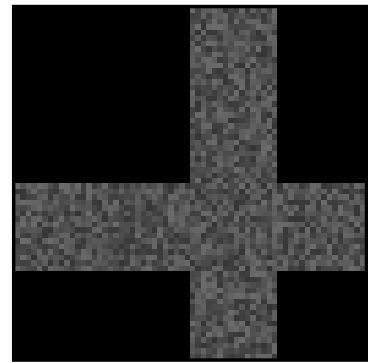

(b)

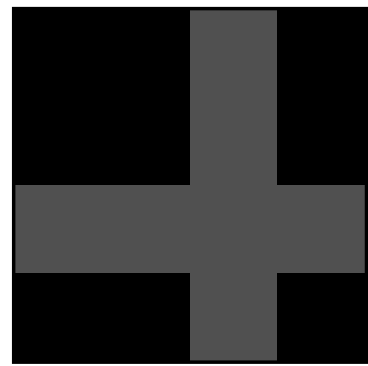

(e)

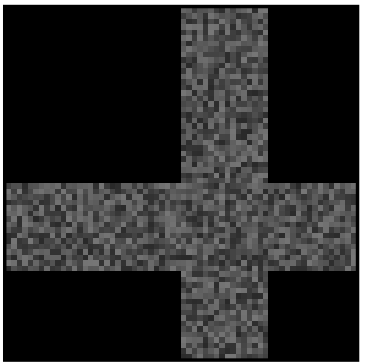

(c)

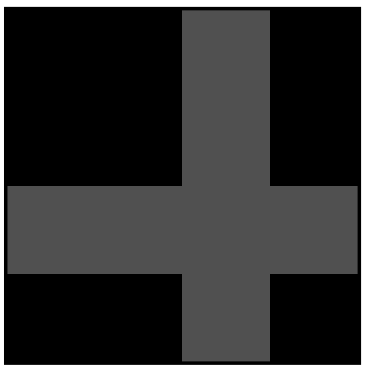

(f)

Figure 7: Results of GA segmentation for images containing (a) - (c) Speckle noise, noisy image with $4.37 \mathrm{~dB}, 3.19 \mathrm{~dB}$ and 2.70 dB SNR. (d) - (f) Output images obtained by GA for images (a) - (c).

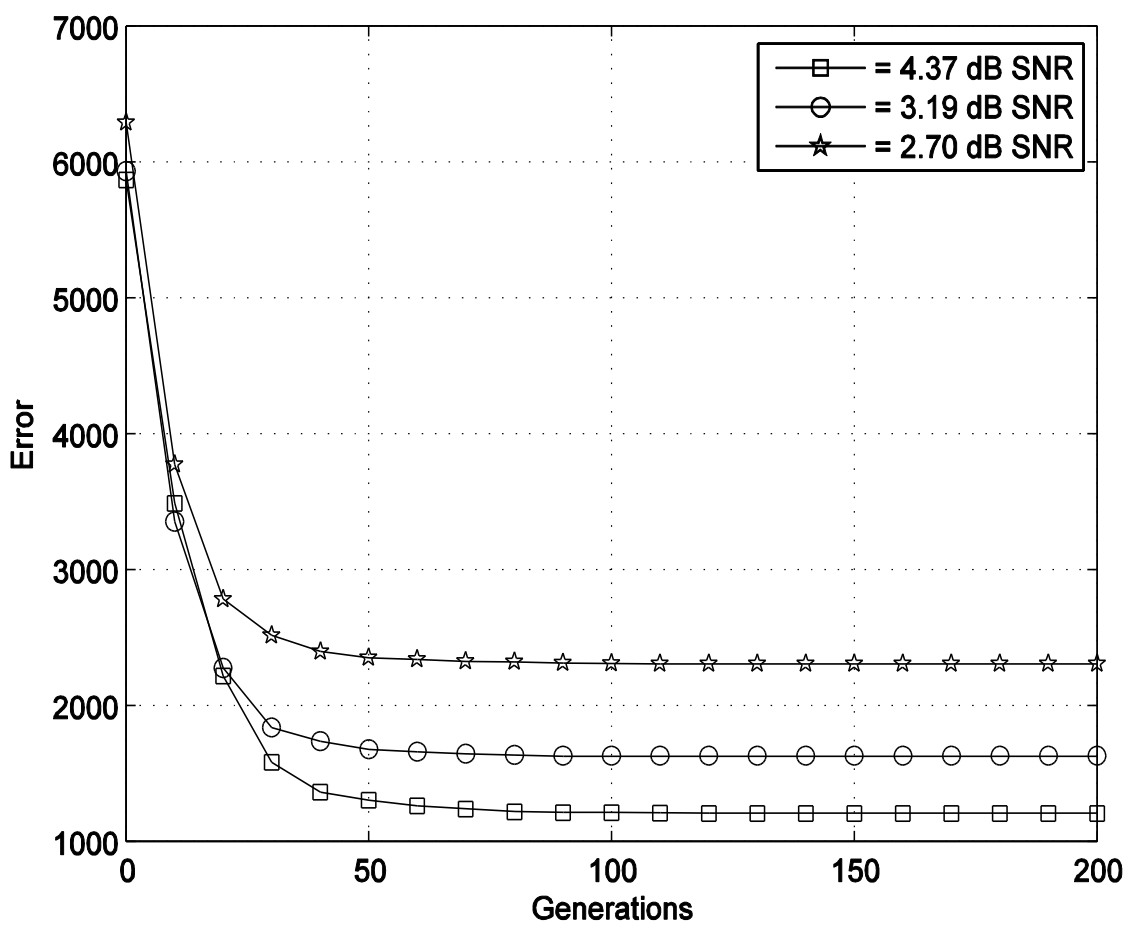

Figure 8: GA convergence graph for segmentation of images containing Speckle noise using $4 \times 4$ image block size.

Table 3: Results of GA segmentation of images containing Speckle noise.

\begin{tabular}{|c|c|c|c|c|c|}
\hline $\begin{array}{c}\text { Sr. } \\
\text { No. }\end{array}$ & $\begin{array}{c}\text { SNR of Input } \\
\text { Image }\end{array}$ & $\begin{array}{c}\text { Population } \\
\text { Size (GA) }\end{array}$ & $\begin{array}{c}\text { Number of } \\
\text { Generations }\end{array}$ & $\begin{array}{c}\text { Misclassified } \\
\text { Pixels }\end{array}$ & $\begin{array}{c}\text { Segmentation } \\
\text { Accuracy (\%) }\end{array}$ \\
\hline 1 & 2.70 & 60 & 200 & 0 & 100.0 \\
\hline 2 & 3.19 & 50 & 200 & 0 & 100.0 \\
\hline 3 & 4.37 & 40 & 200 & 0 & 100.0 \\
\hline
\end{tabular}




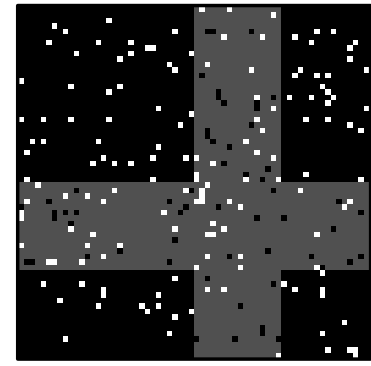

(a)

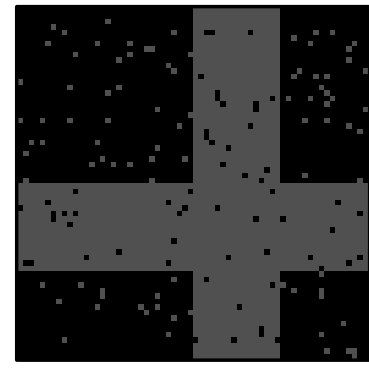

(d)

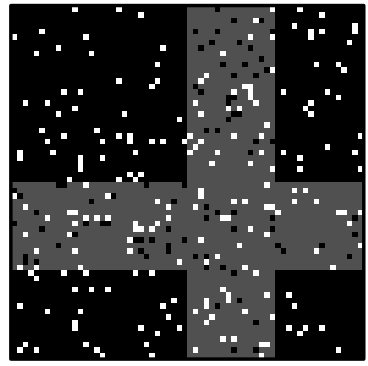

(b)

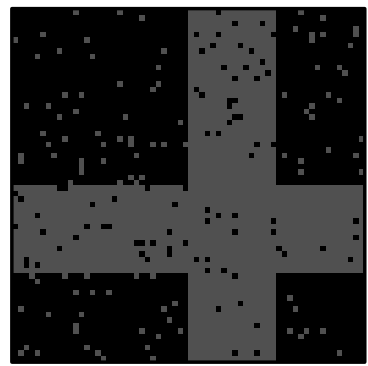

(e)

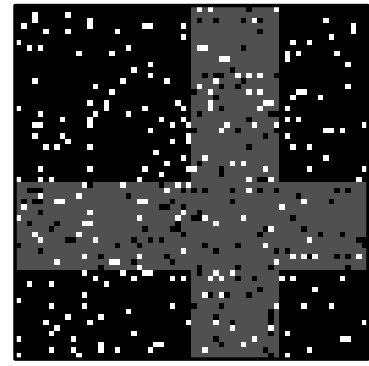

(c)

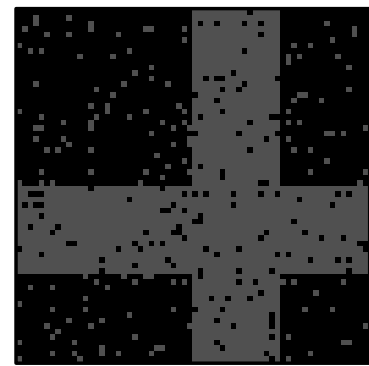

(f)

Figure 9: Results of GA segmentation for images containing (a) - (c) Salt and Pepper noise, noisy image with 11.92 dB, $8.57 \mathrm{~dB}$ and 7.69 dB SNR. (d) - (f) Output images obtained by GA.

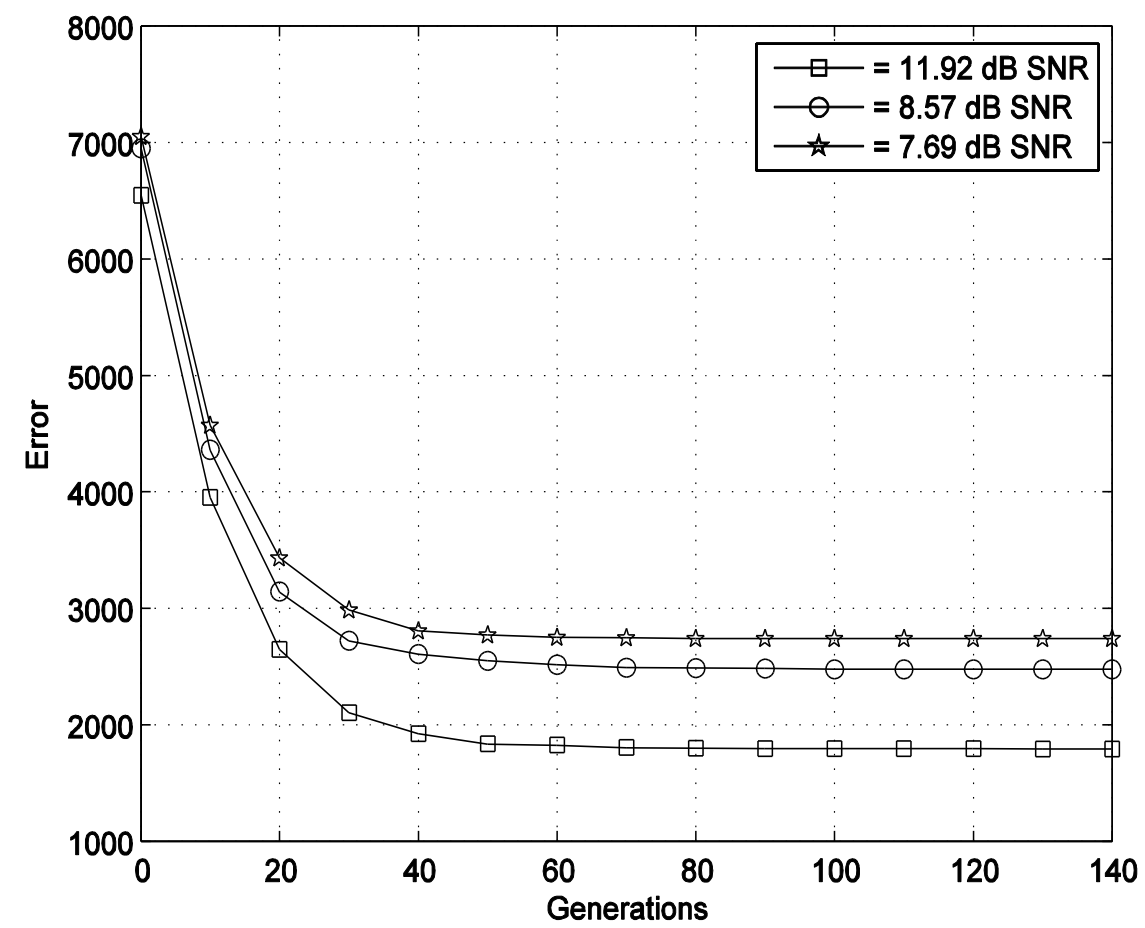

Figure 10: GA convergence graph for segmentation of images containing Salt and Pepper noise using $4 \times 4$ image block size.

Table 4: Results of GA segmentation of images containing Salt and Pepper noise.

\begin{tabular}{|c|c|c|c|c|c|}
\hline $\begin{array}{c}\text { Sr. } \\
\text { No. }\end{array}$ & $\begin{array}{c}\text { SNR of Input } \\
\text { Image }\end{array}$ & $\begin{array}{c}\text { Population } \\
\text { Size (GA) }\end{array}$ & $\begin{array}{c}\text { Number of } \\
\text { Generations }\end{array}$ & $\begin{array}{c}\text { Misclassified } \\
\text { Pixels }\end{array}$ & $\begin{array}{c}\text { Segmentation } \\
\text { Accuracy (\%) }\end{array}$ \\
\hline 1 & 7.69 & 70 & 140 & 269 & 93.4 \\
\hline 2 & 8.57 & 60 & 140 & 187 & 95.4 \\
\hline 3 & 11.92 & 50 & 140 & 148 & 96.3 \\
\hline
\end{tabular}

Difference in Object and Background Intensities: The expected to have a significant effect on the performance of the difference in intensities of the object and background is segmentation algorithm. Hence, the GA is next used for 
segmentation of images having different intensities of object and background. It is also desirable to check the effect of intensity values on GA convergence.

First the GA was applied for the images shown in Figure 11 (a) - (c) which have a pixel difference of 80 pixels in the intensities of the object and background. Three images are used with pixel intensities in the low range, mid range and high range. In particular, the intensities of the background and the object for these images are as (a) 0, 80, (b) 90, 170 and (c) 175,255 . The GA population was set to 100 and image block size of $4 \times 4$ and $8 \times 8$ was used for two GA runs on each image. The other GA parameters were same as before.

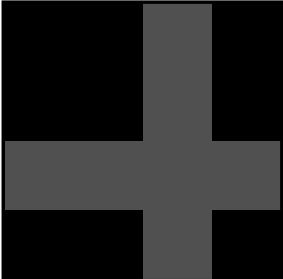

(a)

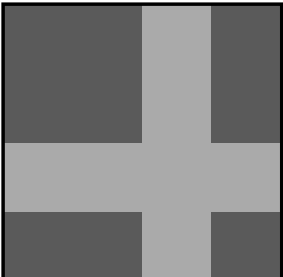

(b)

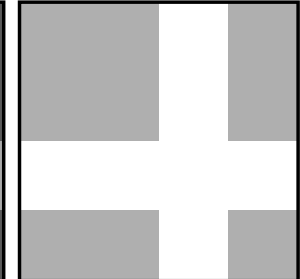

(c)
Figure 11: Input image set.

The results obtained are shown in Table 5 and the convergence graph is shown in Figure 12. It may be observed that there is no much effect of the specific intensity levels on the convergence of GA run. All the GA runs provides correct segmented image i.e. with zero misclassified pixels.

Table 5: Results of GA segmentation of images with pixel intensities in the low range, mid range and high range.

\begin{tabular}{|c|c|c|c|c|c|}
\hline \multirow{2}{*}{$\begin{array}{l}\text { Sr. } \\
\text { No }\end{array}$} & \multirow[t]{2}{*}{ Input Image } & \multicolumn{2}{|c|}{ Using $4 \times 4$ Image Block Size } & \multicolumn{2}{|c|}{ Using $8 \times 8$ Image Block Size } \\
\hline & & $\begin{array}{l}\text { Number of } \\
\text { Generations }\end{array}$ & $\begin{array}{l}\text { Misclassified } \\
\text { Pixels }\end{array}$ & $\begin{array}{l}\text { Number of } \\
\text { Generations }\end{array}$ & $\begin{array}{c}\text { Misclassified } \\
\text { Pixels }\end{array}$ \\
\hline 1 & Figure 11 (a) & 30 & 0 & 400 & 0 \\
\hline 2 & Figure $11(b)$ & 30 & 0 & 400 & 0 \\
\hline 3 & Figure $11(\mathrm{c})$ & 30 & 0 & 400 & 0 \\
\hline
\end{tabular}

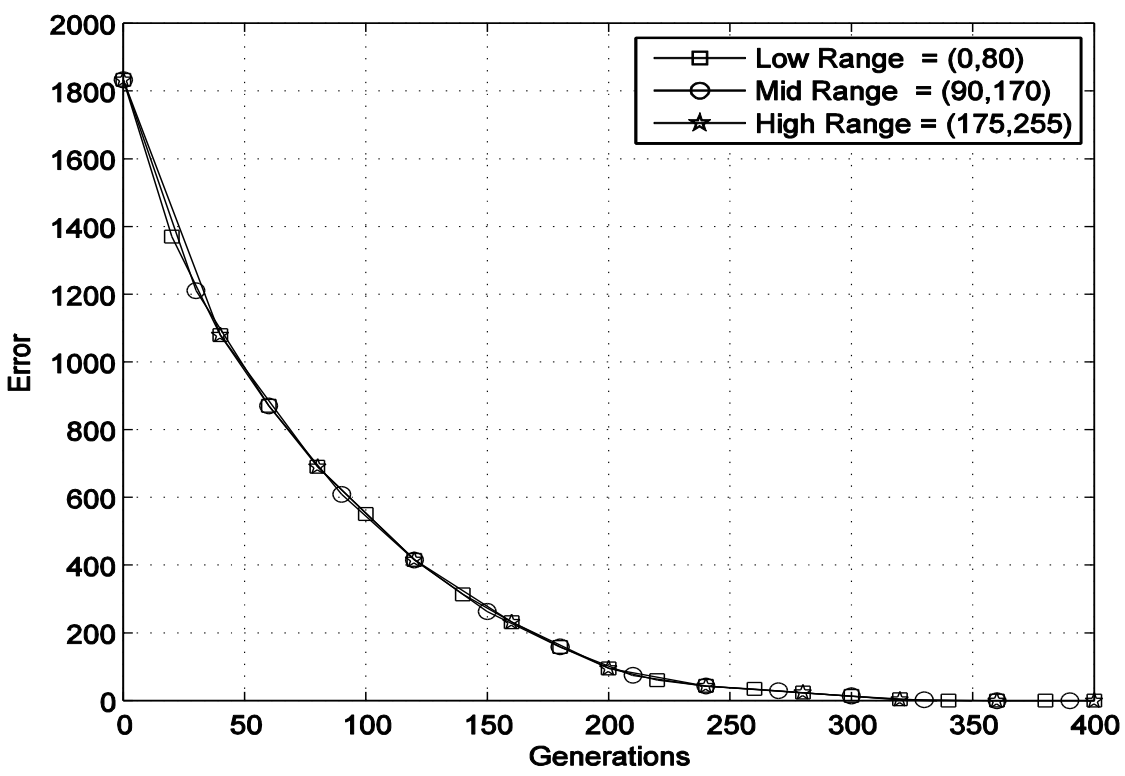

Figure 12: GA convergence graph for segmentation of images shown in Figure 11 (a) - (c) using $8 \times 8$ image block size for pixel intensities in the low range, mid range and high range.

The effect of difference in pixel intensities was considered next. As the specific image intensities did not affect much the segmentation process, the results are obtained only for midintensity region. Five images are considered here with reducing difference as 80,40,20,10 and 5. These images are shown in Figure 13 (a) - (e).

The GA is applied for segmentation of these images using population size of 60 , image block size of $4 \times 4$ and $8 \times 8$. The number of GA generations is set to 1000 . The other GA parameters were same as before.

The results obtained are presented in Figure 13. and the convergence graph is shown in Figure 14. It may be observed from Table 6 that with reducing pixel difference as $80,40,20$, 10 and 5 for $8 \times 8$ image block sizes error is high in output images as compared to image block of size $4 \times 4$. 


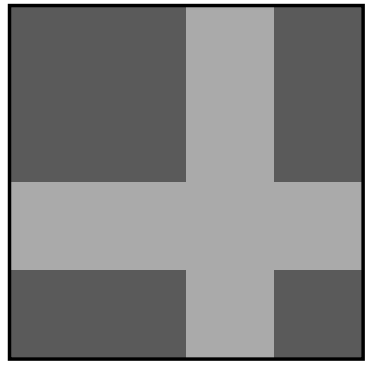

(a)

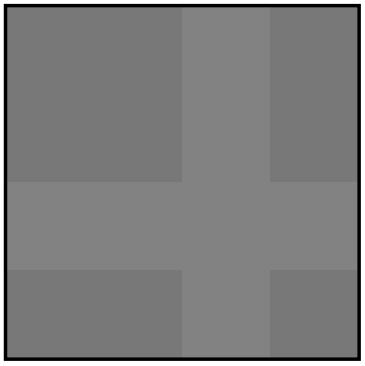

(d)

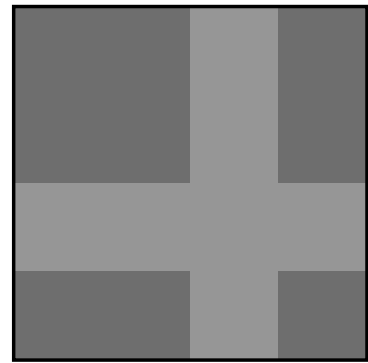

(b)

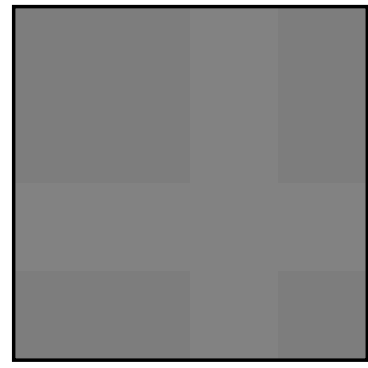

(e)

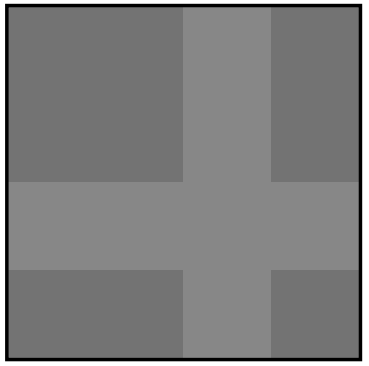

(c)

Figure 13: Input image set for mid-intensity region $(90$ - 170) with reducing difference in object and background intensities as $80,40,20,10$ and 5.

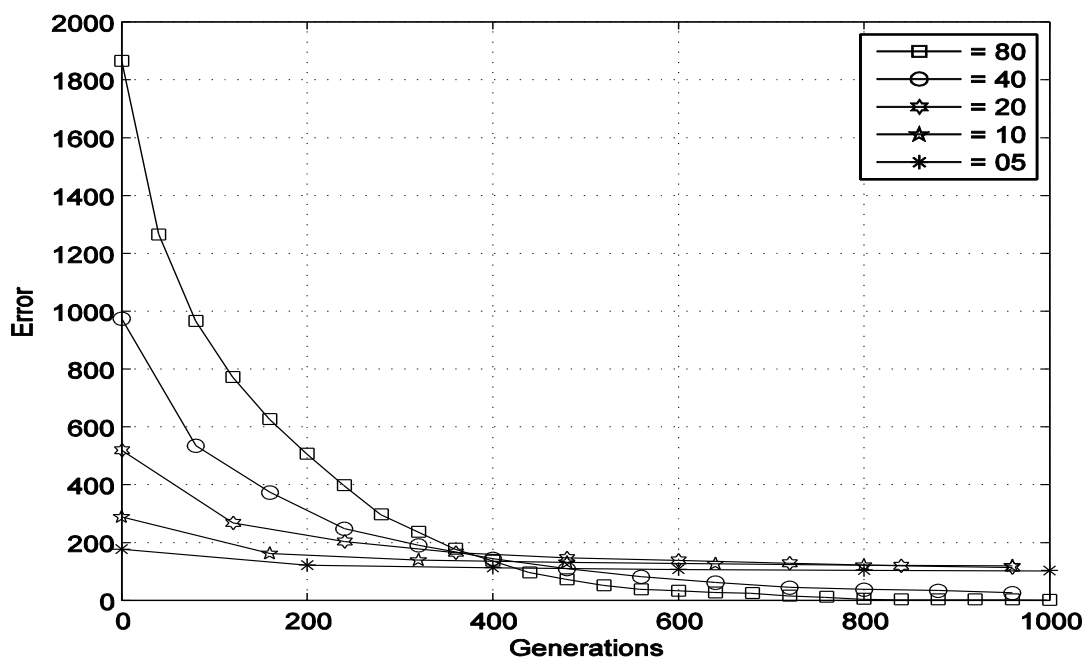

Figure 14: GA convergence graph for segmentation of images shown in Figure 13 (a) - (e) using $8 \times 8$ image block size for midintensity region $(90-170)$ with reducing difference as $80,40,20,10$ and 5.

Table 6: Results of GA segmentation of images have shown in Figure 13 (a) - (e).

\begin{tabular}{|c|c|c|c|c|c|}
\hline $\begin{array}{l}\text { Sr. } \\
\text { No }\end{array}$ & $\begin{array}{l}\text { Input } \\
\text { Image }\end{array}$ & $\begin{array}{c}\text { Pixel } \\
\text { Difference }\end{array}$ & $\begin{array}{l}\text { Background and } \\
\text { Object Intensities }\end{array}$ & \begin{tabular}{|c} 
Misclassified Pixels \\
Using $4 \times 4$ Image Block \\
Size
\end{tabular} & $\begin{array}{c}\text { Misclassified Pixels } \\
\text { Using } 8 \times 8 \text { Image Block } \\
\text { Size }\end{array}$ \\
\hline 1 & Figure 13 (a) & 80 & $(90,170)$ & 0 & 0 \\
\hline 2 & Figure 13 (b) & 40 & $(110,150)$ & 0 & 20 \\
\hline 3 & Figure 13 (c) & 20 & $(115,135)$ & 0 & 181 \\
\hline 4 & Figure $13(d)$ & 10 & $(120,130)$ & 0 & 351 \\
\hline 5 & Figure $13(\mathrm{e})$ & 05 & $(125,130)$ & 0 & 503 \\
\hline
\end{tabular}




\section{CONCLUSION}

A GA has been proposed for the image segmentation of 2-D images. The proposed GA improves in segmentation accuracy with more generations i.e. 80 and larger population sizes. For the crossover rate between 0.50 to 0.70 and mutation rates between 0.01 and 0.08 gives the $100 \%$ pixel classification accuracy. The segmentation accuracy reduces with increases in density level of salt and pepper noise as compared to Speckle noise. The proposed GA gives much better result with block size $4 \times 4$ compared to the $8 \times 8$ and $16 \times 16$ block sizes. It may be observed that reducing pixel difference as $80,40,20$, 10 and 5 for $8 \times 8$ image block sizes error is high in output images as compared to image block of size $4 \times 4$.

Thus the proposed GA-based approach gives us good results for the segmentation of binary images using different image parameters namely the image block size, the difference between the object and background intensities, noise in the image and image size. Also the effect of various GA parameters is considered that include population size, number of GA generations and crossover and mutation rates.

\section{REFERENCES}

[1] C. Bradhurst, W. Boles, and Yin Xiao, "Segmentation of Bone Marrow Stromal Cells in Phase Contrast Microscopy Images," IEEE 23rd International Conference on Image and Vision Computing, pp. 1 - 6, November - 2008.

[2] G. Bosco, "A Genetic Algorithm for Image Segmentation," IEEE Computer Society, 11th International Conference on Image Analysis and Processing, pp. 262 - 266, September - 2001.

[3] B. Bhanu, J. Ming, and L. Sungkee, "Closed-loop Adaptive Image Segmentation," IEEE Conference on Computer Vision and Pattern Recognition, pp. 734 - 735, June - 1991.

[4] R. S. Bichkar and A. K. Ray, "Tomographic Reconstruction of Circular and Elliptical Objects using Genetic Algorithm," IEEE Signal Processing Letters, vol. 5, no. 10, pp. 241 - 251, October - 1998.

[5] M. Haseyama, N. Iwai, and H. Kitajima, "Ccriterionbased Image Segmentation Method with a Genetic Algorithm," IEEE International Symposium on Circuits and Systems, vol. 4, pp. 94 - 97, July - 1999.

[6] R. Cornely and W. Kuklinski, "Application of Genetic Optimization to Medical Image Segmentation," IEEE Conference on Bioengineering, pp. 76 - 79, March 1994.

[7] Y. Horita, T. Murai, and M. Miyahara, "Region Segmentation using k-mean Clustering and Genetic
Algorithms," IEEE International Conference on Image Processing, vol. 4, pp. 1016 - 1020, 1994.

[8] P. Schroeter, J. Vesin, T. Langenberger, and R. Meuli, "Robust Parameter Estimation of Intensity Distributions for Brain Magnetic Resonance Images," IEEE Transactions on Medical Imaging, vol. 71, pp. 172 - 186, April-1998.

[9] L. Cinque, S. Levialdi, G. Pignalberi, and R. Cucchiara, "Optimal Range Segmentation Parameters Through Genetic Algorithms," IEEE $15^{\text {th }}$ International Conference on Pattern Recognition, vol. 1, pp. 474 - 477, September-2000.

[10] B. D. Phulpagar and R. S. Bichkar, "Segmentation of Noisy Binary Images Containing Circular and Elliptical Objects using Genetic Algorithms", International Journal of Computer Applications, vol. 66, no. 22, pp. 1 - 7, March - 2013

[11] D. E. Goldberg, Genetic Algorithm in Search, Optimization and Machine Learning, Pearson, Delhi, 2004, $7^{\text {th }}$ Edition.

[12] R. C. Gonzalez, R. E. Woods, Digital Image Processing, Pearson, Delhi, 2008, $3{ }^{\text {rd }}$ Edition.

[13] A. K. Jain, Fundamental of Digital Image Processing, Pearson, Delhi, 1989, $2^{\text {nd }}$ Edition.

[14] E. Gose, R. C. Johnsonbaugh, Pattern Recognition and

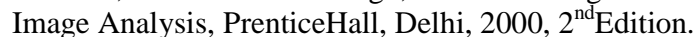

\section{AUTHOR PROFILE}

Dr. Phulpagar B. D. is currently working as Professor in Computer Engineering Department at P. E. S. Modern College of Engineering Pune (India). He graduated from Govt. College of Engineering, Aurangabad affiliated with Dr. Babasaheb Ambedkar Marathwada University, Aurangabad and completed his postgraduate studies at Govt. College of Engineering Pune, affiliated to University of Pune. $\mathrm{He}$ is $\mathrm{Ph}$ D. in Computer Engineering at Shri Guru Gobind Singhji Institute of Engineering and Technology, Nanded, affiliated to Swami Ramanand Teerth Marathwada University Nanded (India).

Dr. Bichkar R. S. is currently working as Professor Department of E\&TC and Dean (R\&D), at G. H. Raisoni College of Engineering and Management, Wagholi, Pune (India). He is $\mathrm{Ph}$. D. from Indian Institute of Technology Kharagpur (India). His areas of research include the application of Genetic Algorithms to Image Processing and DataMining. 